\title{
Splenic-intrahepatic left portal shunt in an adult patient with extrahepatic portal vein obstruction without recurrence after pancreaticoduodenectomy
}

\author{
Satoshi Yamamoto · Yoshinobu Sato • \\ Hiroshi Oya · Hideki Nakatsuka · Takaoki Watanabe • \\ Kazuyasu Takizawa $\cdot$ Katsuyoshi Hatakeyama
}

Received: 2 August 2007 / Accepted: 19 November 2007/Published online: 19 December 2008

(C) Springer 2008

\begin{abstract}
In the last decade, a superior mesenteric-intrahepatic left portal shunt (Rex shunt) has been reported for successful management of extrahepatic portal vein obstruction in children. However, in adults, a mesocaval shunt has been generally performed for the surgical management of extrahepatic portal vein obstruction because of the complexity of the underlying disease and the difficulty of the superior mesenteric-intrahepatic left portal shunt. We herein report an adult patient who was successfully treated by splenic-intrahepatic left portal shunt with an artificial graft (6-mm polytetrafluoroethylene) for complete obstruction of the extrahepatic portal vein following pancreaticoduodenectomy. The shunt procedure not only relieved portal hypertension but also restored hepatic portal flow. In the near future, the Rex shunt should be considered for a beneficial management of extrahepatic portal vein obstruction, even in adults.
\end{abstract}

Keywords Extrahepatic portal vein obstruction .

Splenic-intrahepatic left portal shunt .

Pancreaticoduodenectomy · Mesocaval shunt

\section{Introduction}

Extrahepatic portal vein obstruction (EHPO) is the most common cause of portal hypertension in children, which

S. Yamamoto $(\bowtie) \cdot$ Y. Sato $\cdot$ H. Oya $\cdot$ H. Nakatsuka .

T. Watanabe $\cdot$ K. Takizawa $\cdot$ K. Hatakeyama

Division of Digestive and General Surgery,

Niigata University Graduate School of Medical

and Dental Sciences, 1-757 Asahimachi-dori,

Niigata 951-8510, Japan

e-mail: sugarman@med.niigata-u.ac.jp presents a recurrent hemorrhage from esophagogastric varices and is being treated increasingly by endoscopic injection sclerotherapy (EIS) [1]. Recently, superior mesenteric-intrahepatic left portal shunt (Rex shunt) has been performed in children when endoscopic treatment fails to halt variceal bleeding [2]. However, in adults, the use of mesocaval shunt (MCS) or distal splenorenal shunt (DSRS) has been widely proposed to reduce portal venous pressure and to control variceal bleeding [3]. The Rex shunt has rarely been performed in adults with EHPO, which is associated with severe intraabdominal adhesion resulting from inflammation, trauma, or previous surgery, because of the difficulty of the shunt operation. This report describes an adult case that was successfully treated by splenicintrahepatic left portal shunt with an autograft and an artificial graft for EHPO, which was caused by severe inflammation after pancreaticoduodenectomy.

\section{Case report}

In January 2002, a 61-year-old man underwent pancreaticoduodenectomy in another hospital for pancreatic cancer. Postoperatively, the patient had severe pancreatitis and required several months of treatment before discharge. One and a half year after the surgery, he experienced bleeding from esophageal varices, which was halted by EIS. Thereafter, he suffered repeated episodes of tarry stool, which necessitated red blood cell transfusions.

Upon admission in 2006, a bleeding point was not found by upper gastrointestinal fiberscopy or total colonoscopy. However, the fiberscopy showed severe portal hypertensive gastropathy and colonopathy, including diffused telangiectasia with contact easy bleeding. Abdominal computerassisted tomography (CT scan) and angiography revealed a 
complete obstruction of the extrahepatic portal vein. In the portal phase, the blood flow from the superior mesenteric vein (SMV) drained into the splenic vein and hepatopetal collaterals via the Roux-en Y (Fig. 1a). Although extravasation of the contrast medium into the gastrointestinal tract was not found, the pool of the contrast medium in the jejunal wall surrounding the pancreaticojejunostomy was confirmed in the CT scan. We therefore assumed that the bleeding point was in the Roux-en Y limb of the pancreaticojejunostomy and hepaticojejunostomy.

At first, the nonselective shunt, a mesocaval shunt (Hgraft), was performed, which reduced portal venous pressure from 23.7 to $15.6 \mathrm{mmHg}$ after surgery. A polytetrafluoroethylene (PTFE) graft $5 \mathrm{~mm}$ in a diameter was selected to decrease the incidence of postshunt encephalopathy. However, portosystemic shunt encephalopathy occurred 12 days after surgery. Although serum ammonia levels increased from 88 to $230 \mu \mathrm{g} / \mathrm{dl}$ after surgery, the encephalopathy improved in response to medical treatment. The patient was discharged at 25 days after surgery.
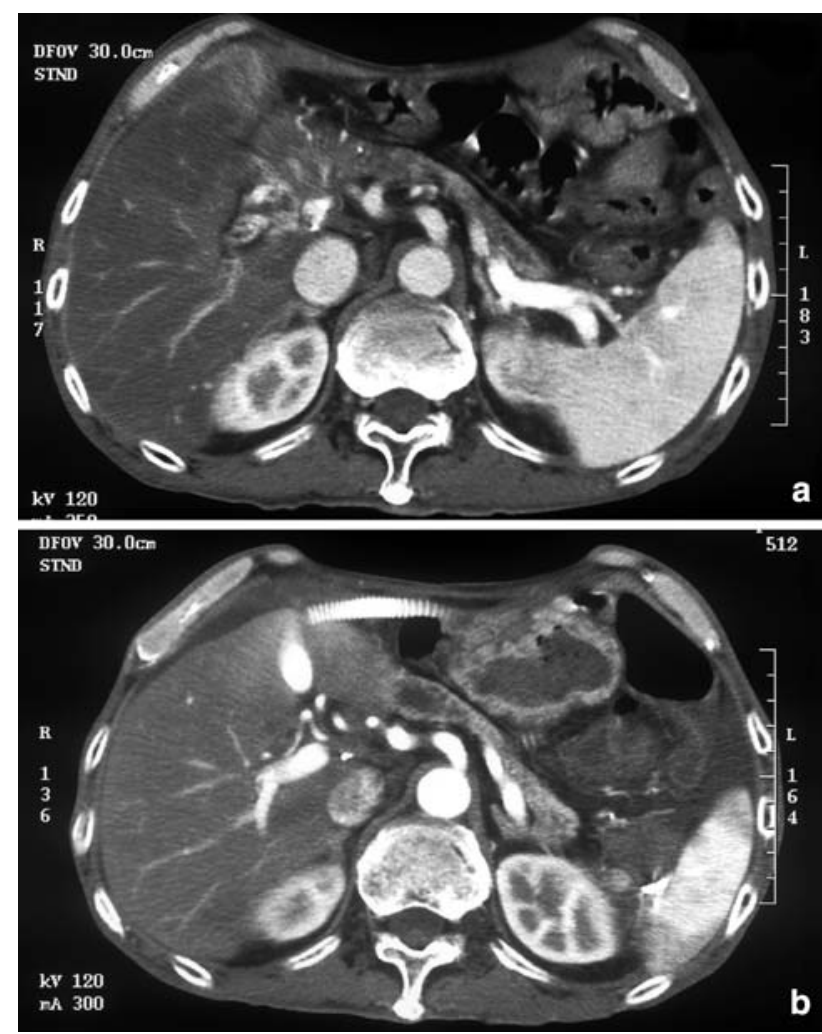

Fig. 1 Abdominal computer-assisted tomography (CT scan) findings before and after splenic-left intrahepatic portal shunt. a CT scan showed a complete obstruction of the portal trunk and hepatopetal collaterals via the Roux-en-Y limb before surgery. b CT scan showed a patent splenic-left intrahepatic portal shunt and disappearance of hepatopetal collaterals via the Roux-en-Y limb at approximately 1 year after surgery. Intrahepatic portal venous flow was remarkably increased
Angiography showed that the patency of the MCS was well maintained 2 months after surgery. Nevertheless, rebleeding occurred from the small intestinal tract. We then carried out a side-to-side anastomosis between the splenic vein and the umbilical portion of the intrahepatic left portal vein to relieve portal hypertension and restore normal hepatic portal flow.

At laparotomy, severe adhesion existed between transverse colon and liver or between the remnant stomach and liver. At first, the transverse colon was dissected from the liver and the remnant stomach. The gastrocolic ligament was opened widely to the hilum of the spleen. The remnant stomach was then retracted both anteriorly and superiorly. Next, the splenic vein was partially separated from the bed of the pancreas body in the back of the pancreaticojejunostomy. Finally, since the umbilical portion of the left intrahepatic portal vein was not exposed because of the tight adhesion of the Roux-en-Y limb to the umbilical fissure, hepatic parenchymal transection was performed toward the umbilical fissure in the division of segments III and IV, allowing exposure of the lower umbilical portion of the intrahepatic left portal vein. A side-to-side splenicintrahepatic left portal shunt was then made with a venous autograft (patient's left external iliac vein) and a PTFE graft $6 \mathrm{~mm}$ in a diameter. The venous autograft was anastomosed end to side to the left portal vein to avoid kinking of the stoma by the hard artificial graft. After that, a PTFE graft $6 \mathrm{~mm}$ in a diameter was interposed between the autograft and the splenic vein (Fig. 2). The portal venous pressure dramatically decreased, from 19.8 to $10.9 \mathrm{mmHg}$, after insertion of the shunt.

The patient has experienced neither gastrointestinal rebleeding nor hepatic encephalopathy for approximately a year after the surgery. The CT scan and the doppler ultrasonography showed a patent splenic-intrahepatic left portal shunt and a disappearance of hepatopetal collaterals via the Roux-en Y limb (Fig. 1b).

\section{Discussion}

EHPO with preserved liver function is an important cause of gastrointestinal hemorrahage accompanying portal hypertension, which may be induced by various causes, including periportal inflammation by cholangitis, pancreatitis, or pancreatobiliary tract surgery [4].

Under these conditions, a portosystemic shunt such as an MCS or inferior mesorenal shunt can offer long-term protection from hemorrhage by decompression of portal venous pressure [3, 5]. However, in EHPO after PD, as in our case, surgical treatment is technically difficult, often involving major blood loss. In addition, the procedure has a high incidence of postshunt encephalopathy [6]. 

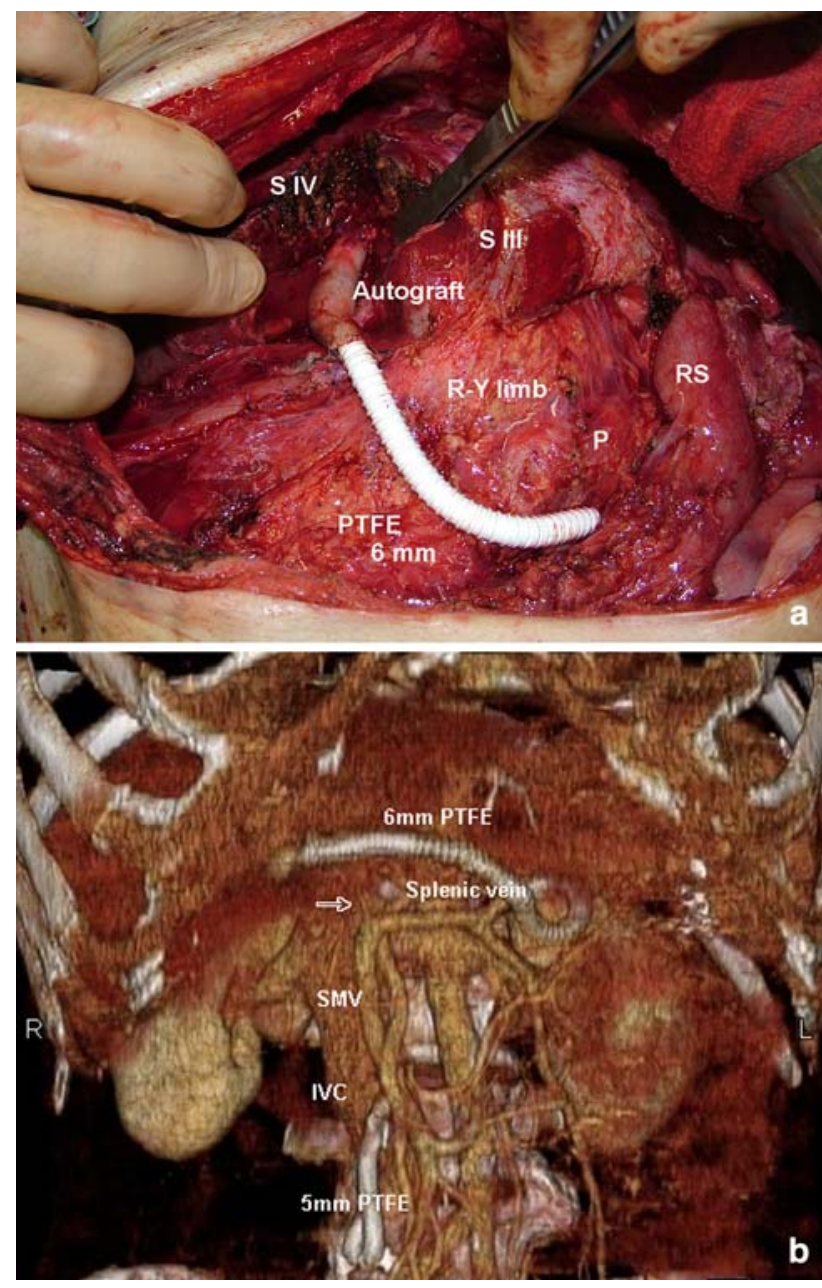

Fig. 2 a Operative findings of splenic-left intrahepatic portal shunt. A side-to-side anastomosis was performed between the splenic vein and the umbilical portion of the left intrahepatic portal vein with an autograft (left external iliac vein) and an artificial graft $(6 \mathrm{~mm}$ in diameter). Extrahepatic portal vein. b Three-dimensional computerassisted tomography findings after splenic-left intrahepatic portal shunt showed a complete obstruction of the extrahepatic portal vein (arrow) and a direct bypassing of extrahepatic portal vein obstruction between the splenic vein and the intrahepatic left portal vein. SMV superior mesenteric vein, IVC inferior vena cava, PTFE polytetrafluoroethylene, $R$-Y limb Roux-en-Y limb, $R S$ Remant stomach, $S$ III Segment III, S IV Segment IV

Although our patient underwent MCS using an artificial graft with a small diameter $(5 \mathrm{~mm})$ to decrease the incidence of postshunt encephalopathy, the portal venous pressure markedly reduced from 23.7 to $15.6 \mathrm{mmHg}$ after surgery. Nevertheless, he experienced intestinal rebleeding and postshunt encephalopathy. Therefore, we designed a direct bypass of the obstructed portal trunk for management of intestinal rebleeding. To our knowledge, our report is the first case to undergo a direct bypassing for EHPO after PD.

Although EIS or portosystemic shunt has been considered as the therapy of choice for management of EHPO, the best choice remains unclear. EIS is more widely available as a first-choice therapy since it is a less traumatic procedure. However, it cannot always provide permanent control of variceal bleeding and cannot control the secondary effects of portal hypertension, including hypersplenism, or hepatic encephalopathy. On the other hand, although portosystemic shunt procedures can offer long-term protection from variceal hemorrhage, the incidence of adverse effects, including a decrease in intrahepatic portal flow or development of postshunt encephalopathy, is reported to be high [6].

In the last decade, there have been a few reports regarding mesenteric-intrahepatic left portal shunt (Rex shunt) for management of EHPO in children. The Rex shunt was first performed by de ville de Goyet in 1992 for a child with portal vein thrombosis that occurred after partial liver transplantation [7]. The report demonstrated that direct bypassing resulted in effective portal decompression and restoration of the hepatic portal flow.

In order to perform Rex shunt, preserved liver function and normal liver structure must be confirmed. Fortunately, our patient had preserved liver function with 7 points of Child-Turcotte-Pugh score although he had pancytopenia. The CT scan showed a patency of the intrahepatic left portal vein. However, we supposed it to be impossible to expose the proximal side of the SMV because of tight adhesion after PD. Therefore, we exposed the proximal splenic vein for the proximal SMV. The proximal splenic vein was anastomosed side to side with the intrahepatic left portal. The patient has experienced neither gastrointestinal rebleeding nor hepatic encephalopathy for approximately a year since the surgery, while continuing with anticoagulation therapy.

However, there are several caveats regarding our shunt procedure for an adult patient who has undergone a previous major surgery. First, the procedure requires a long artificial graft. Accordingly, the patient must temporarily undergo anticoagulation therapy. In addition, the long-term effects of the procedure remain to be elucidated in adults. In the near future, these findings should be confirmed through an accumulation of experience. Second, postoperative adhesion increases the difficulty of such a surgical approach for patients who have previously undergone major abdominal surgery. In these cases, it may be more difficult to expose the umbilical portion of the left intrahepatic portal vein or the splenic vein. In our case, we exposed the umbilical portion by the hepatic parenchymal transection in the division of segments III and IV. The proximal splenic vein was exposed by polite lysis. However, for a case in which the splenic vein cannot be exposed in the back of the pancreas body, the use of the proximal end of the splenic vein after splenectomy may be an alternative for management of EHPO. 
A few authors have very recently reported portal venous stent placement as a useful alternative treatment to MCS for extrahepatic portal vein stenosis because the procedure is less invasive and can restore sufficient portal flow [8]. However, long-term results after stent placement remain unclear. Besides, it may be impossible for patients with complete obstruction of the portal trunk, as in our case.

In conclusion, MCS has thus far been preferred for patients with EHPO as a surgical treatment. However, our experience has demonstrated the beneficial effects of the splenic-intrahepatic left portal shunt for EHPO. Therefore, the shunt procedure should be considered as an alternative management approach to MCS for EHPO in patients who have preserved liver function and normal liver structure, because it is an ideal procedure that not only decreases portal pressure to normal level but also reestablishes adequate portal flow. More experience will be necessary to evaluate the long-term effects of the procedure for EHPO.

\section{References}

1. Zargar SA, Yattoo GN, Javid G, Khan BA, Shah AH, Shah NA, et al. Fifteen-year follow up of endoscopic injection sclerotherapy in children with extrahepatic portal venous obstruction. J Gastroenterol Hepatol. 2004;19:139-45.

2. Ates O, Hakguder G, Olguner M, Secil M, Karaca I, Akgur FM. Mesenterico-left portal bypass for variceal bleeding owing to extrahepatic portal hypertension caused by portal vein thrombosis. J Pediatr Surg. 2006;41:1259-63.

3. Xu CE, Zhang SG, Yu ZH, Li GX, Cao LL, Ruan CL, et al. Combined devasculization and proximal splenorenal shunt: is this a better option than either procedure alone? J Hepatobiliary Pancreat Surg. 2004;11:129-34.

4. Mitsunaga S, Kinoshita T, Kawashima M, Konishi M, Nakagohri $\mathrm{T}$, Takahashi S, et al. Extrahepatic portal vein occlusion without recurrence after pancreaticoduodenectomy and intraoperative radiation therapy. Int J Radiat Oncol Biol Phys. 2006;64:730-5.

5. Yamamoto S, Sato Y, Nakatsuka H, Oya H, Kobayashi T, Hatakeyama K. Beneficial effect of partial portal decompression using the inferior mesenteric vein for intractable gastricesophageal variceal bleeding in patients with liver cirrhosis. World J Surg. 2007;31:1266-71.

6. Sarin SK, Nundy S. Subclinical encephalopathy after portosystemic shunts in patients with non-cirrhotic portal fibrosis. Liver. 1985;5:142-6.

7. de Ville de Goyet J, Clapuyt P, Otte JB. Extrahilar mesenterico-left portal shunt to relieve extrahepatic portal hypertension after partial liver transplant. Transplantation. 1992;53:231-2.

8. Ota S, Suzuki S, Mitsuoka H, Unno N, Inagawa S, Takehara Y, et al. Effect of a portal venous stent for gastrointestinal hemorrhage from jejunal varices caused by portal hypertension after pancreaticoduodenectomy. J Hepatobiliary Pancreat Surg. 2005; 12:88-92. 